\title{
SYNTHESIS AND CHARACTERIZATION OF NANOPOROUS NIO NANOFLAKES SYNTHESIZED USING CHEMICAL BATH DEPOSITION
}

\author{
G. M. Lohar* A. S. Shelke, O. C. Pore, D. V. Rupnavar, C. P. Mane, R.V. Shejwal
}

Lal Bahadur Shastri College of Arts, Science

and Commerce, Satara 415002

Email: gauravlohar24@gmail.com

Communicated : 12.07 .19

Revision :21.07.19 \& 21.08.19

Accepted : 14.09 .19

Published: 30.09 .19

\begin{abstract}
:
In the present work, $\mathrm{NiO}$ nanoflakes are developed using the simple chemical bath deposition (CBD) method. The structural, morphological, and electrochemical properties are investigated for deposited $\mathrm{NiO}$ nanoflakes. The $\mathrm{NiO}$ nanoflakes observed a cubic structure, and $\mathrm{Ni}-\mathrm{O}$ bonds are confirmed the formation of NiO using CBD. The FESEM results confirm the formation of nanoflakes. The nanoflakes deposited at 120 min shows better electrochemical properties compared to other deposited $\mathrm{NiO}$ thin films. In the present study, variation in deposition time has been
\end{abstract} studied.

Keywords: - Nanoflakes, Nickel oxide, chemical bath deposition

\section{INTRODUCTION:}

Environmental pollution and energy consumption have become more and more severe with industrial development. Therefore, dealing with industrial pollution and finding a substitute for traditional energy has become a significant challenge [1-2]. The super capacitors are considered as one of the most promising energy storage devices due to their excellent properties such as fast charge-discharge rate, high power density, low cost, and long cycle life. According to an energy storage mechanism, supercapacitors (SCs) can be divided into electrical double-layer capacitors (EDLCs) and pseudocapacitors [3-4]. Pseudocapacitors materials mainly contain transition metal oxides, hydroxides, sulfides. Among transition metal oxides, $\mathrm{NiO}$ has attracted wide attention due to its high theoretical capacity, wide operating potential window, natural abundance, and environmental compatibility [5-6]. The $\mathrm{NiO}$ is also able to form different nanostructures by changing any chemical as well as a physical parameter. By considering the advantages of $\mathrm{NiO}$ in energy storage devices as well as the need for energy storage devices, many research groups published literature in recent years.

The morphological investigation for improving the supercapacitor is an important task, and a different research group develops recently different nanostructures. Zhang et al. [7] developed $\mathrm{NiO}$ based hierarchical hollow nanofibers for improving the specific capacitance. Also, observed specific capacitance $700 \mathrm{f} / \mathrm{g}$ with 96\% efficiency. Yang [8] et al. synthesized $\mathrm{NiO}$ based flake using chemical bath deposition and studied electrochemical properties in $1 \mathrm{M} \mathrm{KOH}$ and used for an electrochromic application. Das et al. [9] synthesized $\mathrm{NiO}$ nanostructures using the chemical bath deposition and studied structural, optical, and electrical properties. Also, it investigated the effect of thickness on its consequence properties, the three-dimensional nonporous NiOthin films deposited by Liang et al. [10]. For electrochemical supercapacitor and observed high specific capacitance $1776 \mathrm{~F} \mathrm{~g}^{-1}$ in $\mathrm{KOH}$.

By observing literature, it is necessary to develop $\mathrm{NiO}$ based nanostructures and study the electrochemical properties.

In the present work, $\mathrm{NiO}$ nanoflakes are developed using the simple chemical bath deposition (CBD) method. The structural, morphological, and electrochemical properties are investigated for deposited $\mathrm{NiO}$ nanoflakes. 
The $\mathrm{NiO}$ nanoflakes observed a cubic structure, and Ni-O bonds are confirmed the formation of $\mathrm{NiO}$ using CBD. The FESEM results confirm the formation of nanoflakes. The nanoflakes deposited at $120 \mathrm{~min}$ shows better electrochemical properties compared to other deposited thin films. In the present study, variation in deposition time has studied.

\section{EXPERIMENTAL:}

Analytical grade chemicals usedfor the experiment. For the synthesis of $\mathrm{NiO}$ thin films, $0.3 \mathrm{M}$ nickel nitrate was used as a precursor.The $\mathrm{pH}$ of the nickel solution has maintained at 12 using the ammonia solution. The temperature was maintained at $90^{\circ} \mathrm{C}$. The thin films were deposited with three different times, such as 30 , $60 \mathrm{~min}$, and $90 \mathrm{~min}$. The as-deposited thin films were annealed at $450^{\circ} \mathrm{C}$ for 4 hours. For the deposition of $\mathrm{NiO}$, steel substrates were used. The FTIR study has been made using the FTIR from Lamda instruments PVT ltd. The field emission scanning electron microscopic study has been made using the FESEM from Tescan, Pvt. Ltd. The electrochemical study has been made using the biologic Instruments Pvt Ltd.

\section{RESULT AND DISCUSSION:}

\subsection{X-ray diffraction and Fourier transform infrared spectroscopic study -}

Fig. 1 shows the structural investigation of nickel oxide using the XRD and FTIR. Fig. 1 (a) shows the XRD pattern of $\mathrm{NiO}$ thin film, The observed $d$ values matched with stander $d$ values mentioned in JCPDS card 00-047-1049. The Nio thin films show the cubic structure with space group $\mathrm{Fm}-3 \mathrm{~m}$. The values of $\mathrm{a}=\mathrm{b}=\mathrm{c}=4.177$ and $\alpha=\beta=\gamma=90^{\circ}$. The peak at $37.07,43.12,62.80$, 75.36 and 79.40 represents orientation along (111), (200), (220), (311) and (222), respectively. The FTIR spectra is an important characterization tool for finding atomic and molecular boding in nanomaterials. Fig. 1 shows the FTIR spectra of $\mathrm{NiO}$ nanoflowers. The FTIR spectra are recorded for samples A, B, and C. The peak at 557, 627, $1102 \mathrm{~cm}^{-1}$ have belonged to characteristic vibration of Ni-O. The peak near about $3500 \mathrm{~cm}$ ${ }^{1}$ has belonged to the $\mathrm{O}-\mathrm{H}$ group. As a deposition time increases for $\mathrm{NiO}$, change has been observed in the intensity of $\mathrm{NiO}$ peaks [11-12].
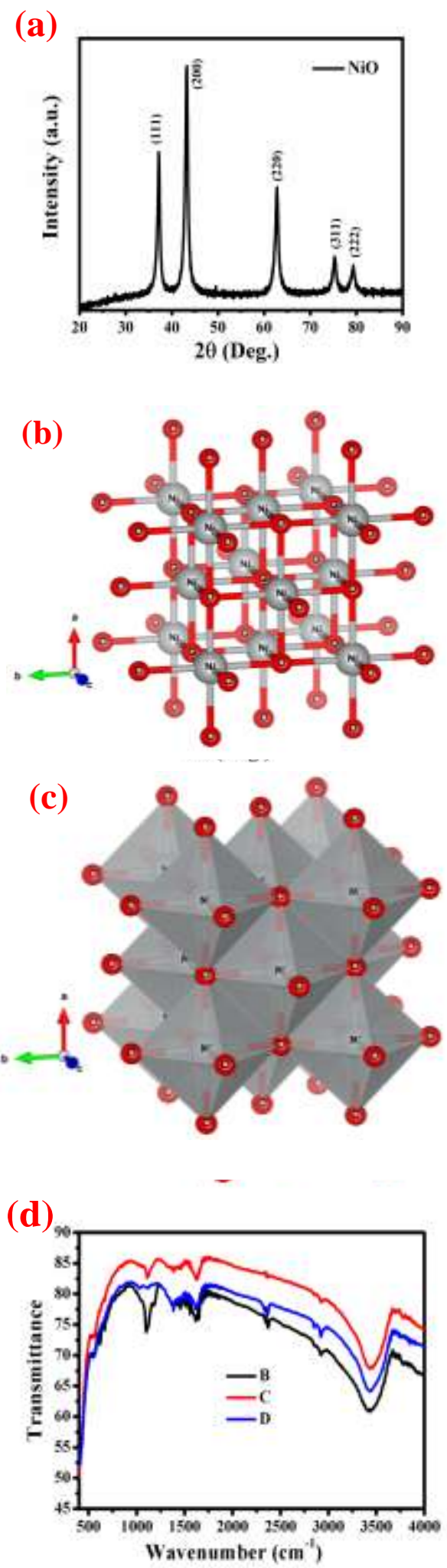

Fig. 1 - FTIR spectra of NiO thin films, (a) XRD pattern of NiO thin film, (b) crystal structure of NiO thin film, (c) Polyhedron of NiO thin film, (d) FTIR of NiO thin film.

3.2. Field Emission Scanning Electron Microscopy - 
Fig. 2 shows the FESEM image of a $\mathrm{NiO}$ thin film. The NiO thin film shows nanoflakes like nature. The FESEM images recorded for a different time, and as time increases, the size of flex was observed to decrease [13]. The nice nanoflakes are observed in Fig. 2. For $60 \mathrm{~min}$ (Fig.2 (a)), the size of flakes is observed to more; also, the distance between two flakes is more as compared to other samples. But as time increases, the distance between two or more flakes observed to be decreased (Fig. 2 (b)). For the $120 \mathrm{~min}$, the size of the flake is very less because ofthe growth of $\mathrm{NiO}$ in free space. The bunch of nanoflakes observed like a flower and observed in Fig. 2 (c).

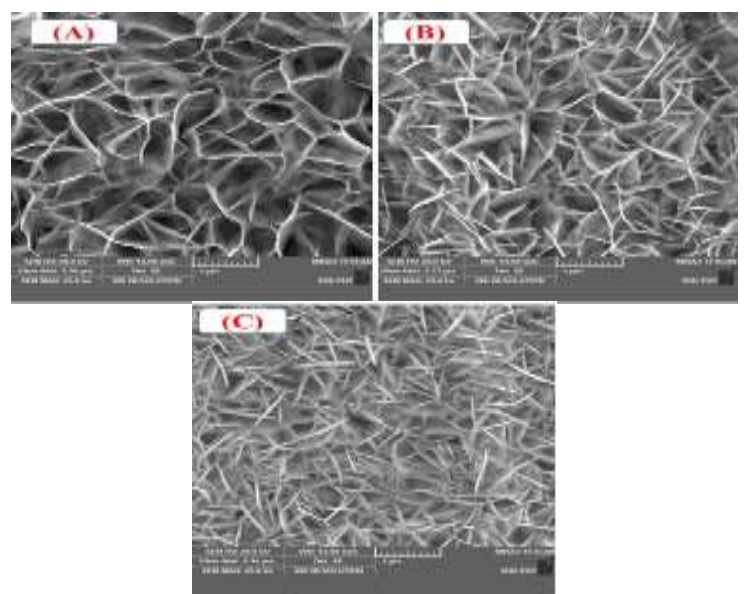

Fig. 2 FESEM images of NiO thin films

\subsection{Electrochemical Impedance spectroscopic study -}

The electrochemical impedance spectroscopy is a tool used to investigate the charge transfer process at the solid-liquid interface [14-15]. Fig. 3 shows the Nyquist plot (Fig.3 (a)) and equivalent circuit (Fig.3(b)) diagram for $\mathrm{NiO}$ thin film. Table. 1 shows the observed parameters from the equivalent circuit diagram. The observed solution resistances are $7.52,5.60$, and $4.68 \Omega$ for 60,90 , and $120 \mathrm{~min}$, respectively. The solution resistance is near about the same for all samples. The charge transfer resistances are $49.05,85$, and $36.8 \Omega$ for 60,90 , and $120 \mathrm{~min}$, respectively. The $\mathrm{NiO}$ thin films deposited at 120 min shows the relatively better charge transfer resistance as compared to other deposited samples. Also, these results are consistent with FESEM results. The thin film deposited at 120 min can be used as efficient supercapacitor material.
Fig. 3 - Electrochemical impedance study of NiO thin film, (a) Nyquist plot NiO Nanoflex, (b) equivalent circuit diagram

Table. 1 Observed parameter from EIS

\begin{tabular}{|l|l|l|l|l|}
\hline $\begin{array}{l}\text { Samples } \\
\text { parameters }\end{array}$ & $\begin{array}{l}\mathbf{R 1} \\
(\boldsymbol{\Omega})\end{array}$ & $\begin{array}{l}\mathbf{C} \\
(\mathbf{\mu F})\end{array}$ & $\begin{array}{l}\mathbf{R 2} \\
(\boldsymbol{\Omega})\end{array}$ & $\begin{array}{l}\mathbf{W} \\
\left(\boldsymbol{\Omega} . \mathbf{s}^{\mathbf{1} / 2}\right)\end{array}$ \\
\hline $\mathbf{3 0} \mathbf{~ m i n}$ & 7.52 & 17.3 & 49.05 & 97.7 \\
\hline $\mathbf{6 0} \mathbf{~ m i n}$ & 5.60 & 13.9 & 85.0 & 130.2 \\
\hline $\mathbf{9 0} \mathbf{~ m i n}$ & 4.68 & 13.8 & 36.8 & 112.0 \\
\hline
\end{tabular}

\subsection{Cyclic voltammetry study -}

The cyclic voltammetry (CV) is a powerful and popular electrochemical technique commonly employed to investigate the reduction and oxidation processes of molecular species [16-17]. The cyclic voltammetry study is shown in Fig. 4 (a, b, c) for $\mathrm{NiO}$ thin films for $60 \mathrm{~min}, 90$ $\mathrm{min}$, and $120 \mathrm{~min}$, respectively. From Fig.4, it is observed that the maximum current and area under the curve is for $120 \mathrm{~min}$. The $\mathrm{NiO}$ sample deposited at 120 min shows the more area under the curve than other deposited samples. It indicates that nanoflakes at $120 \mathrm{~min}$ having more specific capacitance than other deposits.
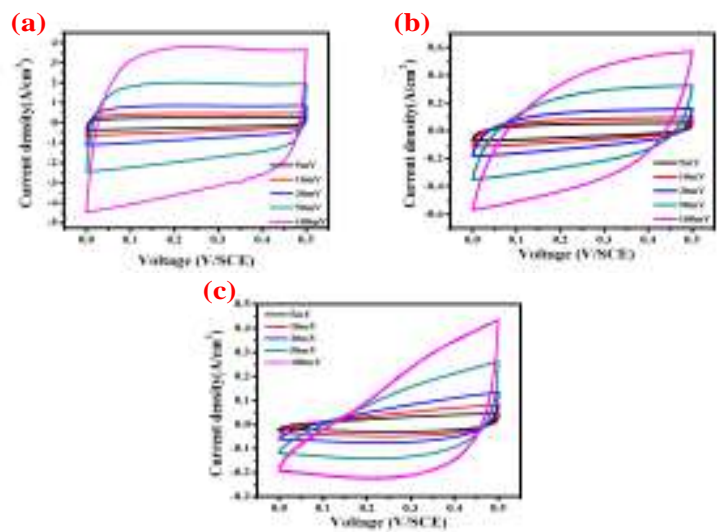

Fig. 4 Cyclic voltammogram for 60,90 , and $120 \mathrm{~min}$.

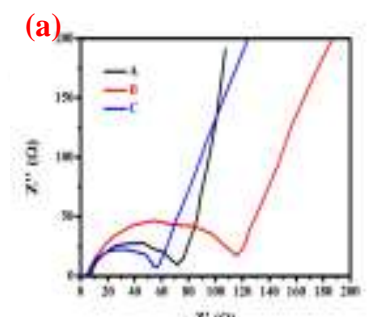

(b)

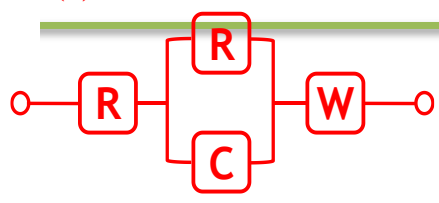




\section{CONCLUSION: -}

In conclusion, nickel oxide thin films have deposited using the chemical bath deposition method. The NiO nanoflakeshave successfully synthesized. The XRD study confirms the formation of $\mathrm{NiO}$ thin film. The peaks at 557, 627, $1102 \mathrm{~cm}^{-1}$ have belonged to the characteristic vibration of Ni-O. The FESEM study shows the thin $\mathrm{NiO}$ having nanoflakes like nature. The electrochemical impedance spectroscopic study exposes the less charge transfer resistance for the thin film deposited at $120 \mathrm{~min}$. The cyclic voltammetry study indicates the more area under the curve for sample deposited at $120 \mathrm{~min}$. In conclusion, $\mathrm{NiO}$ thin films deposited at $120 \mathrm{~min}$ are more useful for supercapacitor applications.

\section{ACKNOWLEDGEMENT:}

Dr. G. M. Lohar thankful to college incubation centre for providing fund research work

\section{REFERENCES:-}

Yirong Zhu, Xiaobo Ji, Ruiming Yin, Zhong liang $\mathrm{Hu}$, Xiao qing Qiu, Zhibin Wu, Yong Liu, Nanorod-assembled $\mathrm{NiCo} 2 \mathrm{O} 4$ hollow microspheres assisted by an ionic liquid as advanced electrode materials for super capacitors, RSC Adv.,2017,7,11123

D. Yu, K. Goh, H. Wang, L. Wei, W. Jiang, Q. Zhang, L. Dai, Y. Chen, Nat. Nanotechnol., 2014, 9, 555-562

P. Simon and Y. Gogotsi, Materials for Electrochemical Capacitors, Nat. Mater., 2008, 7, 845-854

P. J. Hall, M. Mirzaeian, S. I. Fletcher, F. B. Sillars, A. J. R. Rennie, G. O. Shitta-bey, G. Wilson, A. Cruden and R. Carter, Energy Storage in Electrochemical Capacitors: Designing Functional Materials to Improve Performance, Energy Environ. Sci., 2010, 3, 1238-1251

S. Vijayakumar, S. Nagamuthu and G. Muralidharan, Supercapacitor Studies on NiO Nanoflakes Synthesized Through a Microwave Route, ACS Appl. Mater. Interfaces, 2013, 5, 2188-2196

T. Nathan, A. Aziz, A. F. Noor and S. R. S. Prabaharan, Nano-structured $\mathrm{NiO}$ for Electrochemical Capacitors: Synthesis and Electrochemical Properties, J. Solid State Electrochem., 2008, 12, 1003

Min Zhang, Qi Li, Dong Fang, Ismail Alperen Ayhan, Yue Zhou, Lijie Dong, Chuanxi iong, Qing Wang, NiO hierarchical hollow nanofibers as high performance supercapacitor electrodes, RSC Adv., 2015, 5, 96205

Hyeeun Yang, Jung-Hoon Yu, Hyeon JinSeo, Rak Hyun Jeong, Jin-Hyo Boo, Improved electrochromic properties of nanoporous $\mathrm{NiO}$ film by $\mathrm{NiO}$ flake with thickness controlled by aluminum, Applied Surface Science, 461, (2018) 88-92

M.R.Das, A.Mukherjee, P.Mitra, Structural, optical and ac electrical characterization of CBD synthesized $\mathrm{NiO}$ thin films: Influence of thickness, Physica E: Lowdimensional Systems and Nanostructures, 93, 2017, 243-251

Kun Liang,a Xian zhong Tanga and Wencheng $\mathrm{Hu}$, High-performance three-dimensional nanoporous $\mathrm{NiO}$ film as a supercapacitor electrode, J. Mater. Chem., 2012,22, 11062-11067

X.H. Xiaa J.P. Tua J. Zhanga X.L. Wanga W.K. Zhangb H. Huang, Morphology effect on the electrochromic and electrochemical performances of $\mathrm{NiO}$ thin films, Electrochimica Acta

Volume 53, Issue 18, 20 July 2008, Pages 57215724

Yanlong Tian, Zhaoyi Li, Shuliang Dou, Xiang Zhang, Jiaqiang Zhang, Leipeng Zhang, Lili Wang, Xin Zhao, Yao Li, Facile preparation of aligned $\mathrm{NiO}$ nano tube arrays for electrochromic application, Surface and Coatings Technology, 337, 2018, 63-67

Minghua Chen, Jiawei Zhang, Xinhui Xia, Meili Qi, Jinghua Yin, Qingguo Chen, Selfsupported Ni decorated $\mathrm{NiO}$ nano flake arrays as promising cathode materials of hybrid batteries, Materials Research Bulletin76, 2016, 113-117

A. V. Fulari, M. V. Ramana Reddy, S. T. Jadhav, G. S. Ghodake, Dae-Young Kim, G. M. Lohar, TiO2/reduced graphene oxide composite based nano-petals for supercapacitor application: effect of substrate, Journal of Materials Science: Materials in Electronics volume 29, pages 10814-10824(2018)

G. M. Lohar, S. T. Jadhav, B. P. Relekar, R. A. Patil, Y. R. Ma, V. J. Fulari, Optik, 158 (2018) 53-63 